\title{
Review of: "Developing an ethanol utilisation pathway based NADH regeneration system in Escherichia coli"
}

\author{
Mohammad Moradi
}

Potential competing interests: The author(s) declared that no potential competing interests exist.

Regarding the manuscript entitled "Developing an ethanol utilisation pathway based NADH regeneration system in Escherichia coli"

The overall idea behind this paper seems interesting, but there are many points that require careful considerations:

1-the abstract must be summarized. currently, its too long. it also needs to be more focused on the study itself than providing general information that is well known to the researchers in the field. the authors also need to highlight the novelty of their work in the abstract section.

2-kewords need to be chosen more carefully and to the point of this study using MESH could be helpful for such a selection

3-Adequate referencing has not been provided in the introduction section. for example the second paragraph

4- Regarding referencing, the authors must make an effort to provide more updated references from last 10 years. try to avoid the older ones such as 1980

5-Conclusion of the manuscript has the same issue of abstract. try to summarize your work in 1 paragraph 6-The quality of figures are too low. at least $300 \mathrm{dpi}$ resolution is required

7-The aim of your study must be presented at the last sentence of introduction

8- If you use short form of minutes as min, try to write it the same all through the paper. We see it written differently as minutes and mins repeatedly

9- The formula in the material and method has been provided as figure, which makes it look strange. write it again as words than a figure

10- statistical analysis method and used program must be added.

11-P-value must be defined when you compare your results

12-the authors need to state how many times a test was repeated regarding the error bars 13-The graphs provided use different colors for one item. it could be confusing to the readers 14-One major issue regarding this manuscript is that no device output (such as chromatogram) has been provided and its only based on the graphs drawn by authors. device outputs must at least be provided as supplementary information

15- The discussion section is so weak and requires more comparison with similar studies 
